\title{
Skin lesions in a patient with hairy cell leukaemia
}

\author{
Pia Raanani, Michael Thaler, Nathan Keller, Isaac Ben-Bassat
}

A 62-year-old woman was diagnosed as suffering from hairy cell leukaemia, on the basis of bone marrow biopsy results and a positive tartrate-resistant acid phosphatase stain. A single course of 2-chlorodeoxyadenosine was administered as a continuous intravenous infusion over seven days. During the following weeks her complete blood count reached almost normal values except for a lymphopenia of $0.5-1.0 \times 10^{9} / 1$ which persisted during the following months and was attributed to the lympholytic effects of 2-chlorodeoxyadenosine. Bone marrow biopsy six months after treatment showed complete remission of the haematologic disease.

Three months later the patient presented with two new erythaematous nodules, approximately $2 \times 2 \mathrm{~cm}$ each, overlying the dorsal aspect of her right hand (figure 1). Otherwise the physical examination was normal. At that time the complete blood count was normal except for lymphopenia of $0.6 \times 10^{9} / 1$ with an abnormal CD4/CD8 ratio of less than 1.0. The absolute

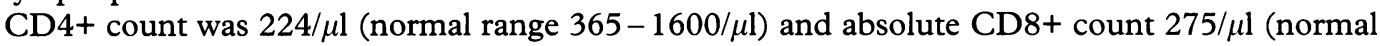
range $270-2400 / \mu \mathrm{l}$ ). Histology of the skin biopsies taken from both lesions is shown in figure 2 . The immunohistochemical stain with a monoclonal anti-human hairy cell leukaemia antibody (clone DBA.44) was negative. Cultures were obtained from both lesions. While the microbiological test results were pending, the patient was treated for a week with clarithromycin for an incidental upper respiratory tract infection. A significant regression in the size of both skin lesions was noted.

The Chaim Sheba Medical Center, Tel-Hashomer and Sackler School of Medicine, Tel-Aviv University, TelHashomer 52621, Israel

The Institute of

Haematology

P Raanani

I Ben-Bassat

Department of

Medicine

M Thaler

Department of Clinical Microbiology

N Keller

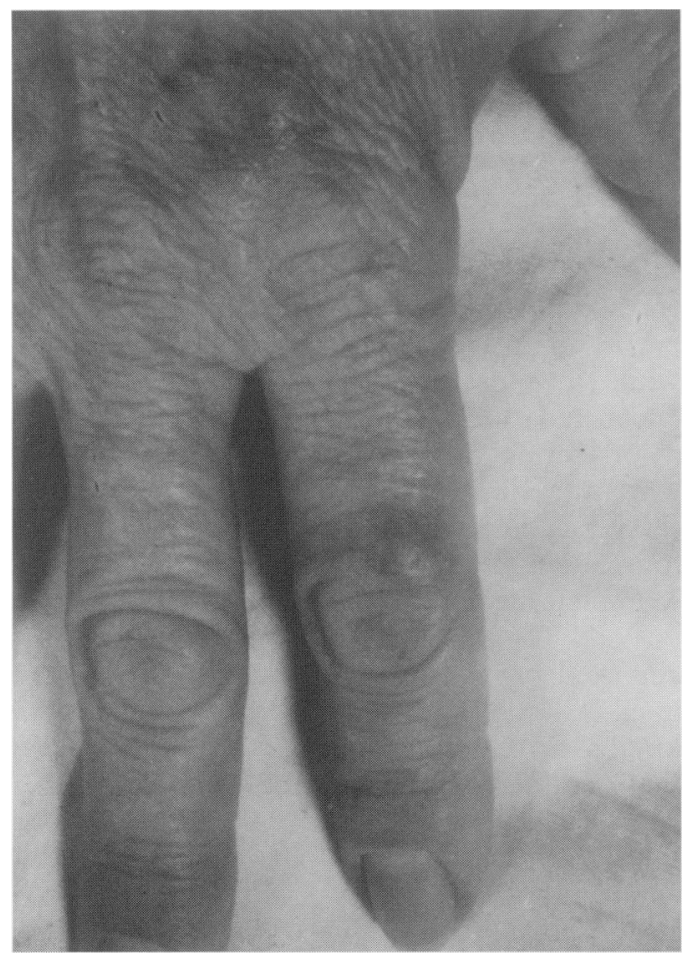

Figure 1 Erythaematous nodules overlying the dorsal aspect of the patient's right hand

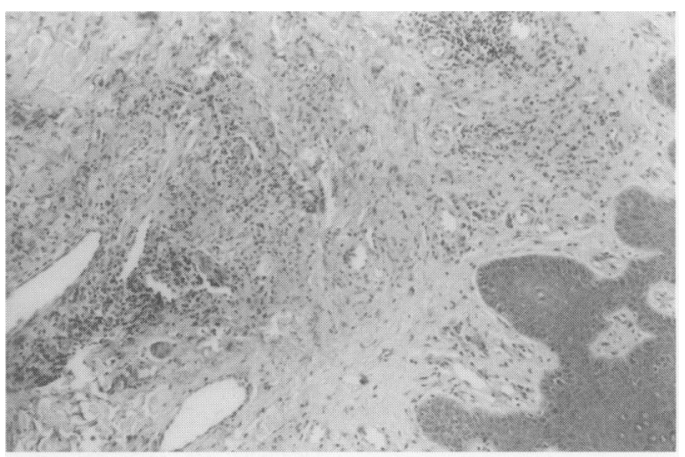

A

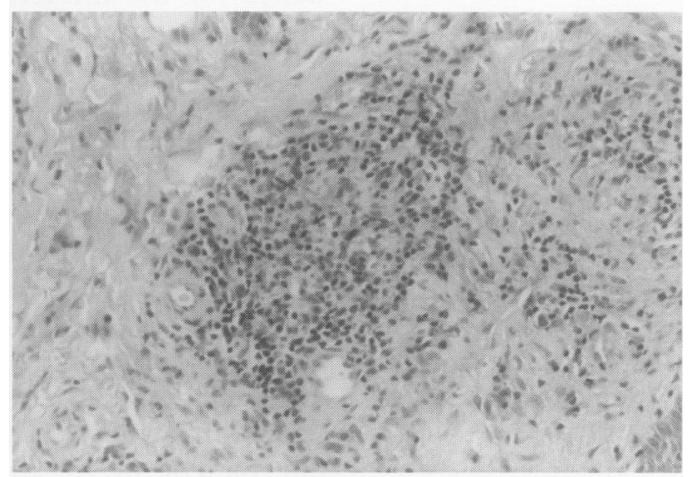

B

Figure 2 (A) Slight hyperplasia of the epidermis; chronic inflammation with giant cells in dermis; (B) granulomata surrounded by hyaline-fibrous tissue $(\mathrm{H}$ \& E)

\section{Questions}

1 What is the pathological diagnosis?

2 What is the most likely pathogen grown in the cultures obtained?

3 What is the treatment of choice in this case? 


\section{Answers}

QUESTION 1

The histology of the skin lesions is granulomatous dermatitis.

QUESTION 2

The most likely pathogen is an atypical Mycobacterium sp. Hairy cell leukaemia patients suffer from mycobacterial infections during the course of their disease. The low CD4 count during the occurrence of the skin lesions might have contributed to the occurrence of a mycobacterial infection. Cultures obtained from both skin lesions indeed grew Mycobacterium kansasii.

\section{QUESTION 3}

New treatments of atypical cutaneous mycobacterioses include the newer macrolides and quinolones. This patient was treated with the combination of rifampicin, clarithromycin and ofloxacin. After one month of treatment the lesions resolved and treatment with the same drug regimen was continued for nine months.

\section{Discussion}

Specific skin lesions occur in approximately $8 \%$ of patients with hairy cell leukaemia. ${ }^{1}$ The possibility of a cutaneous relapse in our patient was ruled out by the histologic results and the negative immunohistochemical stain with the anti-hairy cell leukaemia antibody. Monocytopenia and a defect in monocyte function, Tlymphocyte suppression and cellular immunologic deficiency have been reported in hairy cell leukaemia. All these abnormalities may account for the high incidence of mycobacterial infections (especially kansasii) in this patient population. ${ }^{2}$ However, while in most series mycobacterial infections occurred during the period of disease activity or during the two months following treatment, ${ }^{3}$ the clinical evidence for the infection in our case was only noted nine months after completion of therapy, while the patient was in complete haematological remission.

Several authors have reported on the profound and durable fall in T-cell sub-populations, including an abnormal CD4/CD8 ratio mainly due to failure of CD4+ counts to return to baseline levels, even more than one year after treatment with 2-chlorodeoxyadenosine. A clinical consequence of these alterations is an increased risk of opportunistic infections. ${ }^{4,5}$ It seems that the initiating factor predisposing our patient to $M$ kansasii infection was the

1 Arai E, Ikeda S, Itoh S, Katayama I. Specific skin lesions as the presenting the presenting symptom

2 Mackowiak PA, Demian SE, Sutker WL, et al. Infections in hairy cell leukemia - clinical evidence of a pronounced defect in cell mediated immunity. $A m \mathcal{F}$ Med 1980; 68: 718-24

3 Offerman MK, Golomb HM. Hairy cell leukemia. Curr Prob Cancer 1984; 8, 7-39.

4 Seymour JF, Kurzrock R, Freireich EJ, Estey EH. 2 Chlorodeoxyadenosine induces durable remissions and prolonged suppression of CD4+ lymphocyte counts in patients with hairy cell leukemia. Blood 1994; 83: 2906-11.

\section{Learning points}

- patients treated with purine analogues, even for a short period, have an increased risk of opportunistic infections for a prolonged period

- patients suffering from hairy cell leukaemia and being treated with one course of 2chlorodeoxyadenosine are immunosuppressed by both the disease and the treatment

- the combination of clarithromycin, ofloxacin and rifampicin is an effective regimen for atypical cutaneous mycobacterioses with low toxicity

immunosuppression related to her haematologic disease, further aggravated by the treatment with 2-chlorodeoxyadenosine. This hypothesis is supported by the low CD4/CD8 ratio noted during the occurrence of her cutaneous lesions, with CD4+ counts significantly below the lower normal range.

Our patient retained the ability to respond with a normal delayed-type hypersensitivity reaction as expressed by the histology of granulomatous dermatitis, despite her presumably anergic state. This observation is in concert with previous reports. ${ }^{2} M$ kansasii usually causes disseminated infections in immunocompromised hosts. ${ }^{6,7}$ However, several cases of cutaneous $M$ kansasii infection have been published, usually associated with immunosuppression, skin pathology or contact with contaminated water. ${ }^{8,9}$ Treatment of such infections requires the administration of several antituberculous drugs for prolonged periods. New antibiotics such as the newer macrolides and quinolones have recently been proven effective in these cases. ${ }^{10}$ The favourable clinical cutaneous response to clarithromycin administered for an unrelated cause and the relatively low toxicity of this combined regimen, encouraged us to consider clarithromycin together with rifampicin and ofloxacin as the treatment of choice in our patient. This drug combination indeed resulted in a satisfactory clinical improvement and should be considered in other patients with cutaneous atypical mycobacterioses.

\section{Final diagnosis}

Cutaneous $M$ kansasii infection.

Keywords: hairy cell leukaemia, 2-chlorodeoxyadenosine, Mycobacterium kansasii

5 Juliusson G, Lenkei R, Liliemark J. Flow cytometry of blood and bone marrow cells from patients with hairy cell leukemia. Phenotype of hairy cells and lymphocyte subsets after treatment with 2-chlorodeoxyadenosine. Blood 1994 83: $3672-81$

6 Lillo M, Oreng S, Cernoch P, Harris RL. Pulmonary and disseminated infection due to Mycobacterium kansasii: a decade of experience. Rev Infect Dis 1990; 12: 760-7.

7 Patel R, Roberts GD, Keating MR, Paya CV. Infections due to nontuberculous mycobacteria in kidney, heart and liver transplant recipients. Clin Infect Dis 1994; 19: 263-73. 
8 Beyt BE, Ortbals DW, Santa Cruz DJ, Kobayashi GS, Eisen AZ, Medoff G. Cutaneous mycobacterioses. Analysis of 34 cases with a new classification of the disease. Medicin (Baltimore) 1980; 60: 95-108.

9 Breathnach A, Levell N, Munro C, Natarajan S, Pedler S. Cutaneous Mycobacterium kansasii infection: case report and review. Clin Infect Dis 1995; 20: 812-7.
10 Witzig RS, Franzblau SG. Susceptibility of Mycobacterium kansasii to ofloxacin, sparfloxacin, clarithromycin, azithromycin and fusidic acid: Antimicrob Agents Chemother 1993 $1997-9$.

\title{
Bacteraemia and intestinal obstruction in a 74-year-old man
}

\author{
GK Davis, MJ Brack, MT Hendrickse, DH Roberts
}

A 74-year-old man presented with a two-week history of fatigue, anorexia, upper abdominal pain and pyrexia. There was also a four-day history of constipation. On examination, he had a lowgrade pyrexia $\left(37.8^{\circ} \mathrm{C}\right)$ and an aortic ejection systolic murmur. There was no evidence of cardiac failure. Investigations showed a haemoglobin of $11.0 \mathrm{~g} / \mathrm{dl}$ with a mean corpuscular volume of $69 \mathrm{fl}$ and a significant neutrophilia. Chest X-ray showed cardiomegaly with clear lung fields. Transthoracic echocardiogram showed a calcified aortic valve without any significant stenosis or regurgitation. Twenty-four hours after admission he developed abdominal distension with radiological evidence of large and small bowel dilatation. Sigmoidoscopy was normal up to $20 \mathrm{~cm}$ and he settled on treatment with intravenous cefotaxime, metronidazole and nasogastric drainage. His antibiotic regimen was changed to high-dose intravenous penicillin when Streptococcus sanguis was isolated from his blood cultures. A barium enema examination could not be performed due to recurrent worsening left ventricular failure. A repeat transthoracic echocardiogram was of limited technical quality and showed a thickened, regurgitant aortic valve. He underwent transoesophageal echocardiography (figure) and had an emergency aortic valve replacement. Eight hours postoperatively, while being extubated, he suffered an unheralded cardiac arrest from which he could not be resuscitated.

\author{
Victoria Hospital, \\ Blackpool FY3 8NR, \\ UK \\ Regional Cardiac \\ Centre \\ GK Davis \\ MJ Brack \\ DH Roberts \\ Department of \\ Medicine \\ MT Hendrickse
}

Accepted 27 June 1996

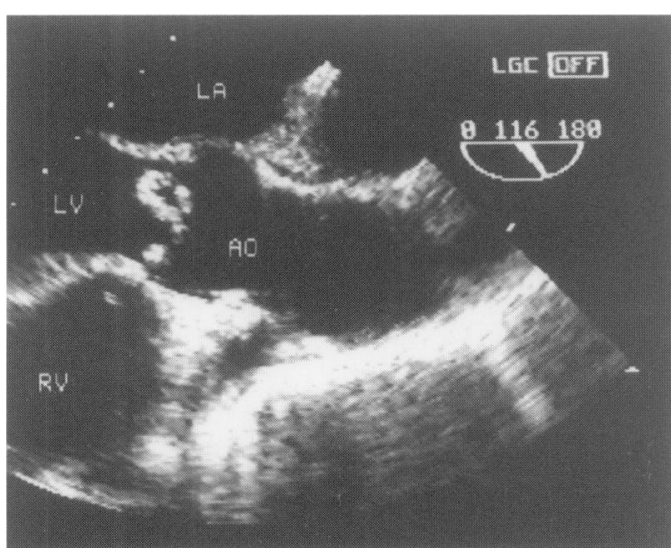

\section{Questions}

1 What is the most likely diagnosis?

2 What does the transoesophageal echocardiogram show?

3 What non-cardiac disorder might have been found at post-mortem?

Figure Transoesophageal echocardiography 